\title{
Comparing the outcome of ileal conduit and transuretero-cutaneostomy urinary diversion after radical cystectomy: a retrospective cohort study
}

\author{
Said Alfin Khalilullah ${ }^{1^{*} \mathbb{O}}$, Untung Tranggono ${ }^{1}$, Ahmad Zulfan Hendri ${ }^{2}$ and R. Danarto ${ }^{2}$
}

\begin{abstract}
Background: Most of the outcomes after radical cystectomy (RC) are directly associated with the type of urinary diversion. This study sets out to evaluate the outcomes of ileal conduit (IC) and transuretero-cutaneostomy (TUC) urinary diversion after RC.
\end{abstract}

Methods: This retrospective study included 52 patients (IC, $n=30 ; T U C, n=22)$ at Dr. Sardjito Hospital between January 2014 and December 2019. The clinical outcomes were compared using Chi-squared tests and independent $t$ tests. Multivariable logistic regression analysis was performed to determine the odds of developing related complications.

Results: Demographically, both groups were similar in terms of age, gender, ASA score, staging, body mass index, and comorbidities. IC was associated with a high incidence of postoperative complications than TUC (56.7\% vs. 27.3\%; $p=0.035)$. Long-term postoperative complications stoma stenosis was more common in the TUC than IC $(p=0.010)$. Multivariate analysis showed TUC was a significant predictor for stoma stenotic with odds ratio of 1.29 (95\% confidence interval, 1.03-1.62; $p=0.006)$. Meanwhile, metabolic change was found higher in IC ( $p=0.047)$. No difference between the rate of required blood transfusion, postoperative ileus, re-operation, and anastomotic stricture in both groups. Operative time $(p=0.000)$ and length of stay $(p=0.002)$ were lower in patients who underwent TUC. The hospitalized cost was also lower in TUC ( $\$ 2311.8 \pm 1448$ for IC vs. $\$ 1844.2 \pm 948.8$ for TUC; $p=0.005$ ). Nonetheless, the follow-up cost was higher in the TUC but not statistically significant. Additionally, there was no difference between the overall satisfaction and diversion-related symptoms scores in both groups. The psychological score was better in IC groups.

Conclusions: Both of these techniques can be an option in a urinary diversion after RC with various advantages and disadvantages. TUC provides reduced complication rates, operative time, shorter length of stay, and hospitalized costs, but IC may reduce postoperative stoma stenosis complications and better psychological function.

Keywords: Bladder cancer, Radical cystectomy, Urinary diversion, Ileal conduit, Transuretero-cutaneostomy

\section{Background}

Radical cystectomy (RC) remains the first option for the treatment of muscle-invasive bladder cancer [1] with a high rate of 5-year disease-free survival [2]. Although

*Correspondence: saidalfink@gmail.com

${ }^{1}$ Division of Urology, Department of Surgery, Dr. Sardjito General

Hospital, Universitas Gadjah Mada, Yogyakarta, Indonesia

Full list of author information is available at the end of the article known for its favorable results, this procedure also has a high morbidity and mortality rate due to surgical 
complications [3]. Most of the complications after RC are directly associated with the type of urinary diversion [4].

In 1852, Simon first described the urinary diversion procedure and Bricker in 1950 popularized the ileal conduit (IC) technique [5]. Since then, this procedure has become popular among surgeons and is considered the first option for urinary diversion procedures [6]. Meanwhile, Johnston described uretero-cutaneostomy in 1963 for a patient with congenital urinary obstruction [7]. Later this procedure, also called transuretero-cutaneostomy (TUC), was used with pelvic malignancy. Both of these techniques have been discussed in several studies regarding advantages and disadvantages [8]. However, several clinical considerations remain problematic, including the time of procedure, postoperative complications, and length of stay.

Additionally, an IC procedure requires an intestinal segment as a urinary diversion, while uretero-cutaneostomy can avoid the complications associated with bowel anastomosis [6]. However, several studies have shown this procedure has a high incidence of ascending infection and stoma stenosis $[6,9]$. Meanwhile, both procedures showed the same result concerning the patients' quality of life [10].

This study evaluated IC and TUC urinary diversion outcome after performing RC in patients with bladder cancer.

\section{Methods}

\subsection{Patient selection and data collection}

In this cohort study, we collected data from 52 patients with bladder cancer who underwent $\mathrm{RC}$ at a single institute between January 2014 and December 2019. This study was registered to the Medical and Health Research Ethics Committee (KE/FK/0608/EC/2020) and approved by the appropriate institutes' ethics committee. The mean followed-up period was 24.8 months (range 12 to 50 ). This cohort was enrolled retrospectively and divided into 30 patients who received IC urinary diversion and 22 patients who underwent TUC. All of the data were collected from medical records. We excluded: (a) patients who received urinary diversion procedures other than IC and TUC, (b) patients with incomplete data in medical records, (c) patients with a laparoscopic procedure, and (d) non-Indonesian ethnic patients. The questionnaire score validated by Asgari was used to measure satisfaction scores [11]. The questionnaire comprises four satisfaction scores covering understand the illness, satisfied the treatment and the operation procedure and spend the life with now condition, and five psychological scores consist of tense, irritable, lonely, anxious, and depressed. It also includes four diversions related to symptom scores of urinary leakage, difficulty managing the stoma, choose another urinary diversion next time, and freely during sleep. The score of all questions ranged from 1 to 4 (not at all to very much).

\subsection{Surgical techniques}

We only collected data from patients who underwent $\mathrm{RC}$ with open surgery by a single operator. The surgeon and patient's choice determined the method of urinary diversion. In the first three years of the study period, we conducted only IC urinary diversion. Both cases underwent RC and pelvic lymph node dissection with a standard transperitoneal method. Urinary diversion using IC was performed using the technique that has previously been described by Bricker [5]. A 15-cm segment of ileum was isolated then uretero-intestinal anastomosis was performed. The ileal segment was brought through the abdominal wall, and a stoma was matured. For the TUC technique, this procedure was described by Johnston in 1963 [7]. The ureters were bilaterally dissected near the ureterovesical junction then cut and spatulated after this point. A 4-Fr ureteral catheter was inserted into the ureter lumen. One of the ureters, to which the other shorter one was attached end-to-side, was connected to the skin. Both techniques used the same type of external urine collection bag.

\subsection{Statistical analysis}

All data analyses were performed using the IBM SPSS 23.00 (IBM Corp., Chicago). Shapiro-Wilk tests were used to analyze the normality of data. When data distribution were skewed, we analyzed the data using a nonparametric test. Differences in baseline or final data between two groups were analyzed using an independent $t$ test (Mann-Whitney $U$ test had skewed data distribution). For categorical data that were non-numeric, analyses used Chi-squared tests. A $P$-value $<0.05$ was considered statistically significant. A multivariant logistic regression model was used for different covariates (age, sex, body mass index (BMI), staging, ASA score, adjuvant chemotherapy, required transfusion, operative time, and urinary diversion) to determine risk of postoperative stoma stenotic.

\section{Results}

The clinical characteristics of patients are shown in Table 1. Both groups were similar in terms of age, gender, ASA score, BMI, staging, and comorbidities. The mean age of patients who underwent IC and TUC was $60.5 \pm 8.9$ years and $59.0 \pm 9.2$ years, respectively. In this study, we performed RC in $7.6 \%$ of patients with metastatic disease due to massive hematuria and decreased hemoglobin levels that were not corrected by blood 
Table 1 Clinical characteristics of the study population

\begin{tabular}{|c|c|c|c|}
\hline & $\mathrm{IC}, N=30$ & TUC, $N=22$ & P \\
\hline Mean $\pm S D$ age, years & $60.5 \pm 8.9$ & $59.0 \pm 9.2$ & 0.551 \\
\hline Gender, $n(\%)$ & & & 0.393 \\
\hline Male & $27(90)$ & $18(81.8)$ & \\
\hline Female & $3(10)$ & $4(18.2)$ & \\
\hline Pathological finding, $n$ (\%) & & & 0.402 \\
\hline Urothelial cell carcinoma & $23(76.7)$ & $20(90.9)$ & \\
\hline Squamous cell carcinoma & $4(13.3)$ & $1(4.5)$ & \\
\hline Adenocarcinoma & $3(10)$ & $1(4.5)$ & \\
\hline Staging, $n(\%)$ & & & 0.167 \\
\hline Organ-confined disease $(\leq \mathrm{pT} 2, \mathrm{pNO})$ & $20(66.7)$ & $8(36.4)$ & \\
\hline Extravesical disease (> pT2 or > pN0) & $7(23.3)$ & $13(59)$ & \\
\hline Metastatic disease & $3(10)$ & $1(4.5)$ & \\
\hline \multicolumn{4}{|l|}{ Comorbidity, n (\%) } \\
\hline Cardiovascular disease & $12(40)$ & $8(36.4)$ & 0.790 \\
\hline Diabetes mellitus & $6(20)$ & $3(13.6)$ & 0.717 \\
\hline Renal insufficiency & $7(23.3)$ & $10(45.4)$ & 0.093 \\
\hline Anemia & $21(70)$ & $14(63.6)$ & 0.629 \\
\hline Hypoalbuminemia & $6(20)$ & $8(36.4)$ & 0.189 \\
\hline \multicolumn{4}{|l|}{ Body mass index } \\
\hline Underweight & $24(80)$ & $15(68.2)$ & 0.389 \\
\hline Norm weight & $6(20)$ & $6(27.3)$ & \\
\hline Overweight & 0 & $1(4.5)$ & \\
\hline ASA score, $n(\%)$ & & & 0.830 \\
\hline 2 & $21(70)$ & $16(72.7)$ & \\
\hline 3 & $9(30)$ & $6(27.3)$ & \\
\hline
\end{tabular}

ASA American Society of Anesthesiologist, IC ileal conduit, TUC transureterocutaneostomy

All statistical analyses in this table used Chi-squared tests

transfusions. All of the patients with advanced disease were continued with adjuvant chemotherapy after surgery.

The overall postoperative complications were significantly higher in IC groups compared with TUC groups (Table 2). Most postoperative complications were stoma stenosis, metabolic changes, wound dehiscence, and anastomotic leak. All of the anastomotic leaks in the IC group were intestine anastomotic, while the TUC group involved ureters end to side anastomotic. One patient in the IC group had intestine anastomotic developed entero-cutaneous fistula one month after surgery and was treated conservatively. During follow-up, serum for acidosis was measure in 23 of 52 (44.2\%). Metabolic acidosis was seen in 10 patients, with $61.5 \%$ in IC groups and $18.2 \%$ in TUC, and was associated with the type of urinary diversion (Table 2). Any of these patients did not need any treatment due to metabolic acidosis.

Four patients showed signs of peritonitis due to intestinal anastomotic leakage and anastomotic end-to-side urethra leakage before the day seventh post-surgery.
Table 2 Postoperative complications in patients who underwent RC on IC and TUC

IC, $N=30 \quad$ TUC, $N=22 \quad P$

\begin{tabular}{|c|c|c|c|}
\hline \multicolumn{4}{|l|}{ Intraoperative complication, $n$ (\%) } \\
\hline Bleeding & $17(56.7)$ & $10(45.4)$ & 0.424 \\
\hline Overall postoperative complications & $17(56.7)$ & $6(27.3)$ & 0.035 \\
\hline \multicolumn{4}{|c|}{ Early postoperative complications, $n(\%)$} \\
\hline Postoperative ileus & $2(6.6)$ & 0 & 0.502 \\
\hline Anastomotic leak & $4(13.3)$ & $1(4.5)$ & 0.381 \\
\hline Sepsis & $2(6.6)$ & $1(4.5)$ & 0.746 \\
\hline Wound dehiscence & $5(16.6)$ & $2(9.1)$ & 0.685 \\
\hline Peritonitis & $3(10)$ & 0 & 0.253 \\
\hline Respiratory distress & $1(3.3)$ & $1(4.5)$ & 0.822 \\
\hline \multicolumn{4}{|c|}{ Late postoperative complications, $n$ (\%) } \\
\hline Stoma stenosis & 0 & $5(22.7)$ & 0.010 \\
\hline Anastomotic stricture & $1(3.3)$ & $1(4.5)$ & 0.822 \\
\hline Entero-cutaneous fistula & $1(3.3)$ & 0 & 1.000 \\
\hline \multicolumn{4}{|l|}{ Biochemical complications, $n(\%)$} \\
\hline Metabolic acidosis * & $8(61.5)$ & $2(18.2)$ & 0.047 \\
\hline Creatinine level increased $>2 \mathrm{mg} / \mathrm{dl}$ & $4(13.3)$ & $3(13.6)$ & 0.957 \\
\hline
\end{tabular}

IC ileal conduit, TUC transuretero-cutaneostomy

All statistical analyses in this table used Chi-squared tests

*Included 23 patients (IC, $n=14$ and TUC, $n=11$ )

All of these patients required emergency re-operation (Table 3). In addition, there were no patients who had complications of stoma stenosis in the IC group, while in the TUC group, $22.7 \%$ of patients had stoma stenosis $(P=0.010)$. The long-term complications of anastomotic stricture were similar in both groups (Table 2).

Patients in the IC groups required more blood transfusion than the TUC group, but the finding was statistically not significant $(P=0.424)$. In patients with TUC urinary diversion, the operative time was significantly lower than in those who underwent IC $(207 \mathrm{~min} \pm 35.7$ vs. $358 \mathrm{~min} \pm 85.5 ; P=0.000$ ), and also the length of stay was significantly shorter in TUC groups (8.5 days \pm 2.1 vs. 13.7 days $\pm 8.0 ; P=0.002$, Table 3 ).

The hospitalized costs were significantly higher in the IC group $(P=0.005)$. Meanwhile, the follow-up cost was higher in the TUC group but not statistically significant.

We modified the questionnaire to evaluate the satisfaction, psychological, and diversion-related symptom scores. The scores are shown in Table 4. A total of 32 patients in this study might be contacted for an interview by phone (IC, $n=19$ and TUC, $n=13$ ). There was no statistical difference in satisfaction and diversion-related symptom scores in both groups. Besides, the psychological score was better in IC groups (Table 4).

We also evaluated patient outcomes during followup to identify the factors that can affect postoperative 
Table 3 Intraoperative and postoperative data according to study groups

\begin{tabular}{llll}
\hline & $I C, N=30$ & TUC, N=22 & $P$ \\
\hline Mean \pm SD operative time, min & $358 \pm 85.5$ & $207 \pm 35.7$ & $0.000^{\mathrm{a}}$ \\
Mean \pm SD intraoperative bleeding, $\mathrm{mL}$ & $698 \pm 311$ & $561 \pm 289$ & $0.113^{\mathrm{a}}$ \\
Requiring blood transfusion, $n(\%)$ & $17(56.7)$ & $10(45.4)$ & $0.424^{\mathrm{b}}$ \\
Postoperative complication rates, $n(\%)$ & $13(43.3)$ & $1(27.3)$ & $0.235^{\mathrm{b}}$ \\
Re-operation rate, $n$ (\%) & $3(10)$ & $8.5 \pm 2.1$ & $0.629^{\mathrm{b}}$ \\
Mean \pm SD length of stay, day & $13.7 \pm 8.0$ & $1844.2 \pm 948.8$ & $0.002^{\mathrm{a}}$ \\
Mean \pm SD hospitalized cost (USD) & $2311.8 \pm 1448$ & $435.0 \pm 529$ & $0.005^{\mathrm{c}}$ \\
Mean \pm SD follow-up cost (USD) & $149.5 \pm 21.0$ & $1(4.5)$ & $0.130^{\mathrm{c}}$ \\
Mortality, early postoperative, $n(\%)$ & $2(6.6)$ & $0.149^{\mathrm{b}}$ \\
\hline
\end{tabular}

IC ileal conduit, TUC transuretero-cutaneostomy, SD standard deviation, USD United States Dollar

${ }^{a}$ Using independent $t$ test analysis

b Using Chi-squared test analysis

c Using Mann-Whitney U test analysis

Table 4 Satisfaction scores post-urinary diversion at > 12-month follow-up in the IC and TUC groups

\begin{tabular}{llcc}
\hline & $I C, N=19$ & TUC, N=13 & $P$ \\
\hline Mean \pm SD satisfaction score & $12.1 \pm 2.77$ & $11.0 \pm 3.21$ & 0.285 \\
Mean \pm SD psychological score & $14.0 \pm 3.77$ & $10.7 \pm 3.32$ & 0.018 \\
Mean \pm SD diversion-related symptom score & $10.3 \pm 2.13$ & $9.9 \pm 2.78$ & 0.613 \\
\hline
\end{tabular}

IC ileal conduit, TUC transuretero-cutaneostomy, SD standard deviation

All statistical analyses in this table used independent $t$ test analysis

Table 5 Multivariable logistic regression analysis for predictors of postoperative stoma stenosis complications ( $n=52$ patients)

\begin{tabular}{lll}
\hline & \multicolumn{2}{l}{$\begin{array}{l}\text { Stoma stenotic-related } \\
\text { complications }\end{array}$} \\
\cline { 2 - 3 } & OR $(95 \% \mathrm{Cl})$ & Pvalue \\
\hline Age ( $\leq 60$ vs $>60$ years & $1.85(0.28-12.1)$ & 0.514 \\
Sex (male vs female) & $0.88(0.80-0.98)$ & 1.000 \\
BMl & $1.22(0.12-12.1)$ & 0.864 \\
Staging & $1.26(1.02-1.55)$ & 0.011 \\
ASA Score & $1.69(0.17-16.5)$ & 0.646 \\
Adjuvant chemotherapy & $0.77(0.61-0.96)$ & 0.010 \\
Required transfusion & $0.20(0.02-1.94)$ & 0.133 \\
Operative time & $0.79(0.64-0.97)$ & 0.011 \\
Urinary diversion & $1.29(1.03-1.62)$ & 0.006 \\
\hline
\end{tabular}

$O R$ odds ratio, $\mathrm{Cl}$ confidence interval, ASA American Society of Anesthesiology, $B M I$ body mass index

stoma stenosis. This study found that the type of urinary diversion and adjuvant chemotherapy were significant predictors of postoperative complication stoma stenosis, with odds ratio (OR) of 1.29 (95\% confidence interval $[\mathrm{CI}], 1.03-1.62 ; P=0.006)$ and $0.77(95 \% \mathrm{CI}$, $0.61-0.96 ; P=0.010$ ), respectively (Table 5 ).

\section{Discussion}

Outcomes of RC have been improving for the last 30 years to become the most common therapeutic strategy for muscle-invasive bladder cancer, with an overall high proportion of 5 years of disease-free survival [12]. Several techniques may be options for urinary diversion after RC, such as IC and TUC [13].

One of the essential considerations in reducing the incidence of mortality and morbidity in patients who undergo RC in bladder cancer is the choice of urine diversion technique $[9,14]$. Unfortunately, there is still often a clinical dilemma in determining the best urine diversion technique until now. Thus, the clinician's decision to select the urinary diversion procedure depends on the operator's preferences.

Recently, as far as we know, only a few studies have reported about $\mathrm{RC}$ in bladder cancer with a primary focus on the type of urinary diversion $[4,13,15,16]$. Although IC urinary diversion is more popular than TUC [14], both procedures follow the same method in using an external bag to collect urine, which can reduce the level of confidence in social interactions [4].

Any form of urinary diversion has advantages and disadvantages. Urinary TUC diversion is simpler because it does not include an intestinal section as a urine source, although this procedure is more associated with 
long-term stoma stenosis $[4,9]$, and often requires lifelong replacement of the ureteric stent [15]. IC urinary diversion has a high rate of complications related to intestinal segments but does not routinely use ureteric stents [4]. In a previous study, the high incidence of bowel-related complications in IC groups supported not to select IC urinary diversion for patient's elderly and poor performance status $[4,17,18]$.

The incidence of intraoperative bleeding and postoperative complications was high in this study. The majority of postoperative complications occurred in the IC group. It should be emphasized that the immediate postoperative complications associated with intestine anastomotic greatly affected the postoperative outcome in the IC groups. Both groups had the same late incidence of postoperative complications: the TUC group was associated with stoma stenosis, thus requiring regular stenting, while the IC groups also have a high incidence of metabolic changes.

Interestingly, all of these patients no required treatment related to metabolic changes. The incidence of postoperative complications is highly dependent on the duration of follow-up. This is seen in a study that indicates complications will rise to $90 \%$ after 15 years [19].

Metabolic changes were the most frequent complication encountered in this study. We found that the incidence of metabolic changes was related to the type of urinary diversion. This result was consistent with the previous one presented in the literature. [20]. Metabolic urinary diversion changes can occur when using the intestinal segment as a urine reservoir [21].

One study conducted by Longo in elderly patients with ASA score $>2$ who underwent radical cystectomy with TUC urinary diversion showed that they experienced shorter operative time, low complications rates, little intensive care monitoring, and less length of stay when compared with IC techniques $(P<0.05)$ [4]. Additionally, some studies also mention cutaneous ureterostomy procedures can be an option in patients with complex comorbidities. Naturally, the ability to minimize operative time and intraoperative bleeding can reduce early postoperative complications $[4,12,15]$. This statement is similar to the findings in our study. We found that the time of operation was shorter (207 $\mathrm{min}$ vs $358 \mathrm{~min}$; $P=0.000)$ and required less blood transfusion $(45.4 \%$ vs 56.7\%; $P=0.424$ ) in patients undergoing RC with TUC urinary diversion.

The length of stay in our study was shorter in the TUC group than the IC urinary diversion group (8.5 days vs. 13.7 days; $P=0.002$ ). Many studies have found a similar result. One study conducted by Deliveliotis in 2005 found that the length of stay in patients undergoing TUC was significantly lower compared with IC urinary diversion with 8.6 and 16.0 days, respectively $(P<0.05)$ [15].

Several studies suggest that IC urinary diversion has similar results regarding patient satisfaction than other procedures [22, 23]. To date, there has been no research explicitly comparing the satisfaction findings after $\mathrm{RC}$ between IC and TUC urinary diversion. In this study, we found that the IC urinary diversion has a better psychological status than TUC. This improvement in psychological scores meant that the IC group had less tense, irritable, lonely, anxious, and depression associated with urinary diversion.

To our knowledge, there are no studies that evaluated the direct hospital-related care cost after performing RC with urinary diversion. We believe, with a reduction in length of stay, the costs will also be reduced. While primarily focused on clinical outcomes, this new concept underlying the TUC urinary diversion also features a significant interplay with health economic considerations. In the future, the perspective on the urinary diversion techniques will rely on additional data collection on the impact of urinary diversion on the patient cost after being hospitalized. Furthermore, patients with an IC urinary diversion experience with no complication of stoma stenotic. In contrast with patients who underwent TUC, $22.7 \%$ of cases have stoma stenosis with OR 1.29 (95\% CI, $1.03-1.62 ; p=0.006)$. Also, there will be additional costs involved if the patient requires regular stenting for postTUC stoma stenotic.

One study conducted by Nieuwenhuijzen also found the same result that the IC urinary diversion had fewer late complications of stoma stenosis [21]. These findings showed that, despite the longer length of stay in the IC groups, this procedure has an advantage in long-term effects. In our opinion, TUC urinary diversion is the simplest procedure with a shorter operative time and also less length of stay. Nevertheless, one disadvantage of TUC was the long-term effects of stoma stenosis.

Our study has several limitations due to the retrospective design and small sample size. However, the data's reliability can be maintained to avoid bias since only one person has done the data collection for a long period. Future studies with high-quality prospective multicenter and randomized control trials are needed to evaluate and confirm TUC urinary diversion's effectiveness compared to IC procedures.

While there are some limitations, for all Indonesian patients, this preliminary study has another strength, which helped preserve the homogeneity of the patients' race. A large number of studies available suggest that the post-RC urinary diversion selection requires different racial and regional approaches. 


\section{Conclusion}

This study showed that the IC and TUC procedures could be an alternative option in the urinary diversion after RC with various benefits in overall patient management. However, both procedure have an disadvantages, the overall postoperative complications was higher in IC, but the incidence of stoma stenosis was more common in TUC. Our study highlights that there is no superiority in both procedures. The decisions depend on clinical conditions, operator experience, and also the patient's preference.

\section{Abbreviations}

IC: Ileal conduit; TUC: Transuretero-cutaneoustomy; RC: Radical cystectomy; ASA: American Society of Anesthesiologist; BMI: Body mass index; SD: Standard deviation; OR: Odds ratio; Cl: Confidence interval; USD: United States Dollar.

\section{Acknowledgements}

The authors acknowledge the medical research unit of Universitas Gadjah Mada for supporting this study.

\section{Authors' contributions}

SAK and UT were involved in study concept and design. SAK and AZH were involved in data acquisition. SAK was involved in drafting of manuscript. SAK, UT and RD were involved in critical revision of the manuscript. All authors have read and approved the manuscript.

\section{Funding}

None.

\section{Availability of data and materials}

The datasets used and/or analyses during the current study are available from the corresponding author on reasonable request.

\section{Declarations}

\section{Ethics approval and consent to participate}

This study was registered with the ethical clearances of Universitas Gadjah Mada, Medical and Health Research Ethics Committee (KE/FK/0608/EC/2020), and approved by the appropriate institutes' ethics committee. Consent to participate was not applicable as this was a retrospective chart review.

\section{Consent for publication}

Not applicable.

\section{Competing interests}

The authors declare that they have no competing interests.

\section{Author details}

${ }^{1}$ Division of Urology, Department of Surgery, Dr. Sardjito General Hospital, Universitas Gadjah Mada, Yogyakarta, Indonesia. ${ }^{2}$ Division of Urology Oncology, Department of Surgery, Dr. Sardjito General Hospital, Universitas Gadjah Mada, Yogyakarta, Indonesia.

Received: 22 December 2020 Accepted: 29 March 2021

Published online: 07 April 2021

\section{References}

1. Yai FA, KassoufW (2009) Radical cystectomy is the treatment of choice for invasive bladder cancer. Can UrolAssoc J 39(5):409-412
2. Kilciler M, Bedir S, Emedir F et al (2006) Comparison of ileal conduit and transureteroureterostomy with ureterocutaneostomy urinary diversion. Urol Int 77:245

3. Guzzo TJ, Vaughn DJ (2016) Management of metastatic and invasive bladder cancer. In: Wein AJ, Kavoussi LR, Partin AW, Peters CA (eds) CampbellWalsh urology, 11th edn. WB Saunders, Philadelphia, p 2223

4. Longo N, Imbimbo C, Fusco F et al (2016) Complications and quality of life in elderly patients with several comorbidities undergoing cutaneous ureterostomy with single stoma or ileal conduit after radical cystectomy. BJU Int 118:521

5. Bricker EM (1950) Bladder substitution after pelvic evisceration. Surg Clin N Am 30:1511-1521

6. Kızılay F, Şimşir A, Cüreklibatır I et al (2018) Long-term outcomes of patients who underwent ureterocutaneostomy. Bullet Uroonco 17:54-58

7. Johnston JH (1963) Temporary cutaneous ureterostomy in the management of advanced congenital urinary obstruction. Arch Dis Child 38:161-166

8. Stein RS, Hohenfellner M, Pahernik S, et al (2012) Urinary diversionapproach and consequences. Dtsch Arztebl Int 617-22

9. Witjes JA, Bruins M, Cathomas R, et al (2019) Muscle-invasive and metastatic bladder cancer. In: EAU Guidelines

10. Rangarajan K, Somani BK (2019) Trends in quality of live reporting for radical cystectomy and urinary diversion over the last four decades: a systematic review of the literature. Arab J Urol 17(3):181-194

11. Asgari MA, Safarinejad MR, Shakhssalim N, Soleimani M, Shahabi A, Amini E (2013) Quality of life after radical cystectomy for bladder cancer in men with an ileal conduit or continent urinary diversion: a comparative study. Urol Ann 5(3):190-196

12. Miller DC, Taub DA, Dunn RL et al (2003) The impact of co-morbid disease on cancer control and survival following radical cystectomy. J Urol 169:105-109

13. Mucciardi G, Macchione L, Galı A et al (2015) Quality of life and overall survival in high risk patients after radical cystectomy with a simple urinary derivation. Cir Esp 93:368-374

14. Dahl DM (2016) Use of intestinal segments in urinary diversion. In: Wein AJ, Kavoussi LR, Partin AW, Peters CA (eds) Campbell-Walsh Urology, 11 th edn. WB Saunders, Philadelphia, pp 2281-2315

15. Deliveliotis C, Papatsoris A, Chrisofos M et al (2005) Urinary diversion in high-risk elderly patients: modiied cutaneous ureterostomy or ileal conduit? Urol 66(2):299-304

16. Siddiqui KM, Izawa Jl (2016) Ileal conduit: standard urinary diversion for elderly patients undergoing radical cystectomy. World J Urol 34:19-24

17. Wuethrich PY, Vidal A, Burkhard FC (2016) There is a place for radical cystectomy and urinary diversion, including orthotopic bladder substitution, in patients aged 75 and older: results of a retrospective observational analysis from a high-volume center. Urol Oncol 34(2):58.e19-27

18. Suzuki K, Hinata N, Inoue T, Nakamura I, Nakano Y, Fujisawa M (2020) Comparison of the perioperative and post-operative outcomes of ileal conduit and cutaneous ureterostomy: a propensity score-matched analysis. Urol Int 104:48-54

19. Madersbacher S, Schmidt J, Eberle JM et al (2003) Long-term outcome of ileal conduit diversion. J Urol 169:985-990

20. Studer UE, Burkhard FC, Schumacher M et al (2006) Twenty years experience with an ileal orthotopic low pressure bladder substitute-lessons to be learned. J Urol 176:161-166

21. Nieuwenhuijzen JA, de Vries RR, Bex A et al (2008) Urinary diversions after cystectomy: the association of clinical factors, complications and functional results of four different diversions. Eur Urol 53:834

22. Philip J, Manikandan R, Venugopal S, Desouza J, Javlé PM (2009) Orthotopic neobladder versus ileal conduit urinary diversion after cystectomy: a quality-of-life based comparison. Ann R Coll Surg Engl 91:565-569

23. Svare J, Walter S, Kvist Kristensen J, Lund F (1985) Ileal conduit urinary diversion: early and late complications. Eur Urol 11:83-86

\section{Publisher's Note}

Springer Nature remains neutral with regard to jurisdictional claims in published maps and institutional affiliations. 